\title{
Failure mechanism analysis of asphalt-aggregate systems subjected to direct shear loading
}

\author{
Mansheng Dong $\mathbb{D} \cdot$ Yanhai Hao $\cdot$ Chao Zhang $\cdot$ Linglin Li
}

Received: 20 April 2017/Accepted: 14 August 2017/Published online: 31 August 2017

(C) The Author(s) 2017. This article is an open access publication

\begin{abstract}
In order to investigate the mechanical behavior of asphalt-aggregate systems subjected to direct shear loading and reveal the shear failure mechanism, four groups of direct shear tests were conducted on composite specimens under different experimental conditions with a self-manufactured direct shear test apparatus at $25{ }^{\circ} \mathrm{C}$. Comparative studies were conducted to evaluate the effects of stone surface treatment, asphalt film thickness and loading rate on the shear mechanical behavior of asphaltaggregate specimens. Results showed that two kinds of the complete stress-displacement curves, including the general single-peak curve and the first-known double-peak curve, were clearly observed for each condition. In addition, the internal failure mechanisms were analyzed based on qualitative and quantitative methods. It can be concluded that the potential failure modes of the direct shear test include adhesive failure at the asphalt-aggregate interface and cohesive failure within the asphalt film. The research results enhance understanding of the shear mechanical behavior and failure mechanism of asphalt mixture, and also provide a reference for the interfacial failure.
\end{abstract}

M. Dong $(\varangle) \cdot$ Y. Hao · C. Zhang $\cdot$ L. Li

School of Automotive and Transportation Engineering, Hefei University of Technology, Hefei 230009, People's Republic of China e-mail: dongms@hfut.edu.cn
Keywords Asphalt-aggregate $\cdot$ Interface $\cdot$ Shear test - Stress-displacement curve $\cdot$ Adhesion $\cdot$ Cohesion

\section{Introduction}

Asphalt mixture has been widely used in highways, airport and bridge pavement constructions. When the vehicles run on the asphalt pavements at a constant speed, the strain and the stress inside the structural interlayers have no obvious changes. However, several cases of slippage cracking and permanent deformation appear at the poor interface where the shear stress exceeds the shear resistance of asphalt mixture, or in the situations where the vehicles frequently brake and turn sharply [1]. In fact, the typical destruction in terms of rutting, peeling, and cracking due to bond weakness are often reported currently, and these irreversible structural and functional deficiencies in the pavements significantly decrease the driving comfort and service capacity of asphalt pavements. Therefore, understanding the mechanism and characterization of the shearing process through experimentation and analyses is an indispensable step for improving road performance and service life.

It is well known that bond conditions between asphalt mixture directly influence road performance. In general, the rheological properties of asphalt mixture are the basis for a better understanding of 
the mechanical behavior of asphalt pavements. During the research of the rheological properties of asphalt materials, one must be clear about the temperature, bonding condition and other influence factors, because the mechanical behavior which is susceptible to internal bonding condition and experiment process shows elasticity, viscoelasticity, viscoplasticity in stages [2-4]. Numerous studies [5-7] prove that the physical properties and mechanical performance of asphalt mixtures are directly dominated by the different manufacturing methods and morphological features at mesoscale level, such as shapes, distribution, asphalt content and void ratio. Furthermore, a number of studies have been conducted to measure and evaluate the adhesive and cohesive properties of the asphalt-aggregate combinations from experimental aspects using pull-off testing [8-10]. These experimental studies have provided acceptable and reasonable results for further understanding adhesive and cohesive properties of asphalt-aggregate systems.

Many researches have found that three factors are responsible for the failure of asphalt mixture, including strength reduction of aggregate particles, cohesive loss within asphalt film and breakdown of adhesive bonding between asphalt and aggregate [8-13]. Cohesive failure is characterized by the separation of molecules within the asphalt film. Adhesive failure is related to the separation of the asphalt coating from the aggregate. In addition, fundamental theories of the adhesive bond of asphalt-aggregate systems including mechanical theory, thermodynamic theory, physicochemical theory, and weak boundary theory have been proposed. Based on the irreversible thermodynamics theory, Zhu et al. [14] provided a viscoelasticviscoplastic damage constitutive model for asphalt mixtures. Liu et al. [15] showed that limestone aggregates could perform better than granite aggregates for moisture resistance and also suggested that the physico-chemical properties of aggregates may play a fundamental and more significant role in the generation of moisture damage than bitumen properties. Mo et al. [16] concluded that the adhesive zone between stone and mortar tended to be the weakest link by comparing the experimental results in the interface region.

Adhesive failure between asphalt and aggregate particles are only part of the causes. Research efforts have been made to investigate the influence of interfacial bonding conditions on the mechanical properties of asphalt mixture. Interfacial bonding in the adhesive zone due to stone surface morphology and asphalt film thickness often have a significant influence on the failure mechanism [16-18]. Therefore, more comprehensive and accurate knowledge of the interface is necessary to better understand the asphalt-aggregate systems, and some significant work, such as shearing fracture mechanism, needs to be done.

The primary objective of this paper is to compare and evaluate the shear characteristics and the failure mechanisms of asphalt-aggregate systems through direct shear tests and statistical analysis. The direct shear tests were conducted under four different experimental conditions at $25{ }^{\circ} \mathrm{C}$. The results obtained include the shear strengths, two types of shear stressdisplacement curves, failure envelopes, and failure modes of asphalt-aggregate systems.

\section{Direct shear test}

The fundamentals of the experiment consist of processing the sandwiched specimens that are cylindrical, $\varnothing 19 \times 80 \mathrm{~mm}^{2}$, and shearing the specimen components in the universal testing machine (WDW1) after maintaining them for $24 \mathrm{~h}$ at room temperature, as shown in Fig. 1. The following sections describe the direct shear test conducted and the results collected.

\subsection{Materials and specimens}

In this study, limestone and SBS modified asphalt were employed. The limestone was obtained from Huangshan Mountain in the south of Anhui province in China. Limestone belongs to carbonate rocks, its main composition is $\mathrm{CaCO}_{3}$, and apparent relative density is $2.473 \mathrm{~g} / \mathrm{cm}^{3}$. Thus, limestone aggregate with high alkalinity used in the direct shear test can perform better bond with acidic asphalt. The SBS modified asphalt is widely adopted in Chinese asphalt pavement engineering. The specimen components were fabricated with the same raw materials including limestone aggregate and SBS modified asphalt with the physical properties shown in Table 1.

More specially, the desired asphalt film of the asphalt-aggregate specimen depends on the fabrication stress, which is set on the specimen by a special 




Fig. 1 Apparatus for the direct shear test

Table 1 Physical properties of SBS modified asphalt

\begin{tabular}{lll}
\hline Physical properties of SBS modified asphalt & Unit & Test result \\
\hline Penetration $\left(100 \mathrm{~g}, 5 \mathrm{~s}, 25^{\circ} \mathrm{C}\right)$ & $0.1 \mathrm{~mm}$ & 53 \\
Softening point & ${ }^{\circ} \mathrm{C}$ & 86 \\
Ductility $\left(5 \mathrm{~mm} / \mathrm{min}, 5{ }^{\circ} \mathrm{C}\right)$ & $\mathrm{cm}$ & 36 \\
Density $\left(15^{\circ} \mathrm{C}\right)$ & $\mathrm{g} / \mathrm{m}^{3}$ & 1.029 \\
\hline
\end{tabular}

clamp holder during the fabrication process. The holder has two clamps: the lower clamp is fixed with the holder base and the upper clamp moves up and down vertically with controlled displacements. The specimen preparation consists of the following steps:

1. Surface pre-treatment The limestone is firstly sawed into slices with the thickness of approximate $40 \mathrm{~mm}$. To obtain a relatively certain roughness, one side of the stone slice is polished (or sandblasted) with a surface treatment apparatus. The roughness of the polished surface is 37.9 um while the other sandblasted surface is $56.0 \mathrm{um}$, which can be examined with a surface roughmeter.

2. Core drilling The limestone slices are drilled for the cylindrical stone columns. Then all stone columns $\left(\varnothing 19 \times 40 \mathrm{~mm}^{2}\right)$ are washed in boiling distilled water for $15 \mathrm{~min}$ to remove any dust on the stone surfaces, and dry them in an oven at $80{ }^{\circ} \mathrm{C}$ for $4 \mathrm{~h}$.
3. Pre-heating the aggregate and the bitumen Stone columns and asphalt are placed in an oven at $160{ }^{\circ} \mathrm{C}$ for $1 \mathrm{~h}$.

4. Fabricating the cylindrical sandwiched specimens A pair of stone columns with the same surface are fastened in the lower clamp and the upper clamp of the holder, respectively. Then, a drop of hot asphalt is placed evenly on the upper surface of the lower stone column and then the columns are pressed together quickly to form a good stone-asphalt bond. Emphasizing that the clamp holder can make the two columns parallel and centered well.

5. Setting fabrication stress A gentle pressure of $89.9 \mathrm{kPa}$ (or $22.5 \mathrm{kPa}$ ) is applied on the upper clamp of the holder to control the thickness of the asphalt layer by a fabrication stress bar. As the upper clamp of the holder would provide a pressure of $2.3 \mathrm{kPa}$, the total pressure loaded on the sandwiched specimen is $92.2 \mathrm{kPa}$ (or 
$24.8 \mathrm{kPa}$ ) during the fabrication process. After $15 \mathrm{~min}$, the pressure is removed from the clamping holder.

6. Conservation After cooling down for about an hour, the sandwiched specimens are taken out from the clamp holder and stored in a room at approximately $25^{\circ} \mathrm{C}$ for $24 \mathrm{~h}$. The excess adhesive asphalt at the edges of the specimen should be trimmed with a heated knife.

\subsection{Experimental method}

The apparatus for direct shear test shown in Fig. 1, is designed to match a microcomputer control electronic universal testing machine (WDW-1) and equipped with an environmental chamber. The environmental chamber can maintain temperatures ranging from -40 to $120{ }^{\circ} \mathrm{C}$ with an error within $\pm 0.5{ }^{\circ} \mathrm{C}$. The shear box that is fixed in the environmental chamber, consists of a loading block, a clamping device, and a guide rail. And the details of the shear box are enlarged in Fig. 1. Similar apparatus [19] has been employed in former researches to investigate the shear characteristics of interface between two different materials. The loading block is fixed on the loading head of WDW-1, while the guide rail is fastened to the base of WDW-1. The clamping device is fixed on the guide rail and can be moved only along the guide rail to meet requirement of the test. There is a gap about $1 \mathrm{~mm}$ between the right face of the loading block and the left face of the clamping device, and the asphalt film of the sandwiched specimen is placed in a center position of the narrow gap. The right part of specimen is fastened by the clamping device, while the left part is suspended. The weight of the suspended part is about $20 \mathrm{~g}$, and it can be neglected in the mechanical analysis [5, 19].

At the beginning of the test, specimens are kept in an environmental chamber for at least $4 \mathrm{~h}$ to equilibrate to the targeted temperature. During the shear test, the applied load and displacement response of the sandwiched specimen are captured automatically by the microcomputer, and the experimental data measured could be used to determine the stress-displacement curve.

In the factorial design, three condition variables were considered: stone surface, asphalt film thickness as well as loading rate. According to previous researches $[2,20,21]$, the similar surface types and loading rates were applied. The asphalt film thickness is determined by the fabrication stress applied to the stone columns during preliminary fabricating specimen. The tests were designed and conducted under four experimental conditions in terms of experimental group (EG), control group of surface treatment (CGST), control group of fabrication stress (CGFS) and control group of loading rate (CGLR) and the details of the experimental conditions are shown in Table 2. Considering the viscoelasticity of asphalt, the test temperature was set at $25^{\circ} \mathrm{C}$.

Twenty-two direct shear tests were performed on the replicate specimens for each condition. In order to verify experimental repeatability, twenty specimen tests were picked out for analyzing test results of each group. In another word, the findings of eighty specimen tests are summarized in this paper.

\section{Results and discussion}

A shear load $\mathrm{F}$ is loaded on the specimens by the shear loading block at a specific loading rate of $5 \mathrm{~mm} / \mathrm{min}$ (or $9 \mathrm{~mm} / \mathrm{min}$ ), and the vertical displacement of the left half of specimen is recorded by the microcomputer. The interface shear strength (or peak shear stress) can be calculated as follows [5, 19]:

$\tau_{\max }=\frac{F_{\max }}{A}$

where $\tau_{\max }$ is the interface shear strength, $F_{\max }$ is the maximal shear load applied to specimen, and $A$ is the cross-sectional area of specimen.

In addition, residual shearing phenomenon was clearly observed in which the vertical displacement continued increasing while the shear stress kept at a constant value. Figures 2, 3, 4, 5, where $R$ is defined as replicate specimen, present the interface shear strengths, residual shear strengths, and corresponding vertical displacements of all the experimental specimens, as well as the failure modes and two types of shear stress-displacement curves obtained from the direct shear tests.

\subsection{Characteristics of the shear stress- displacement curves}

The shear mechanical behavior of asphalt-aggregate specimens can be characterized by the complete 
Table 2 Experimental conditions for the direct shear test

\begin{tabular}{llll}
\hline Experimental condition & Surface type & Fabrication stress $(\mathrm{KPa})$ & Loading rate $(\mathrm{mm} / \mathrm{min})$ \\
\hline EG & Polished surface & 92.2 & 5 \\
CGST & Sandblasted surface & 92.2 & 5 \\
CGFS & Polished surface & 24.8 & 5 \\
CGLR & Polished surface & 92.2 & 9 \\
\hline
\end{tabular}

(a)

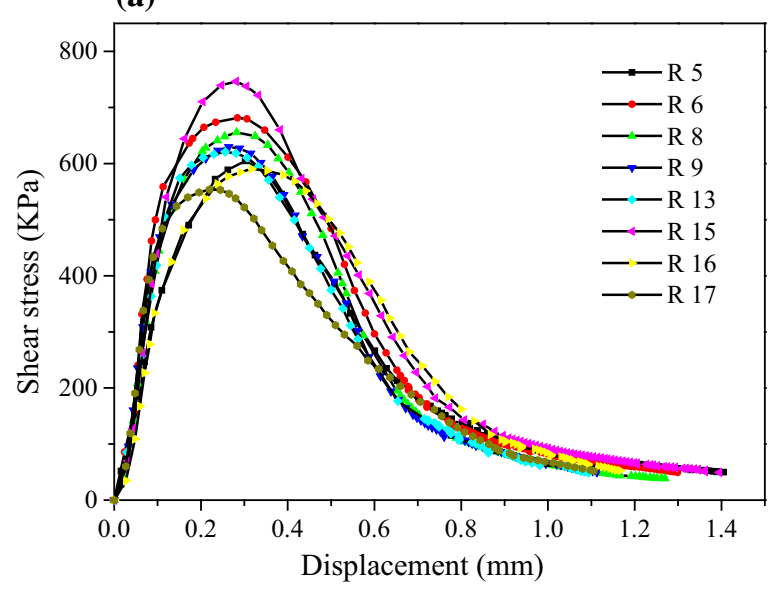

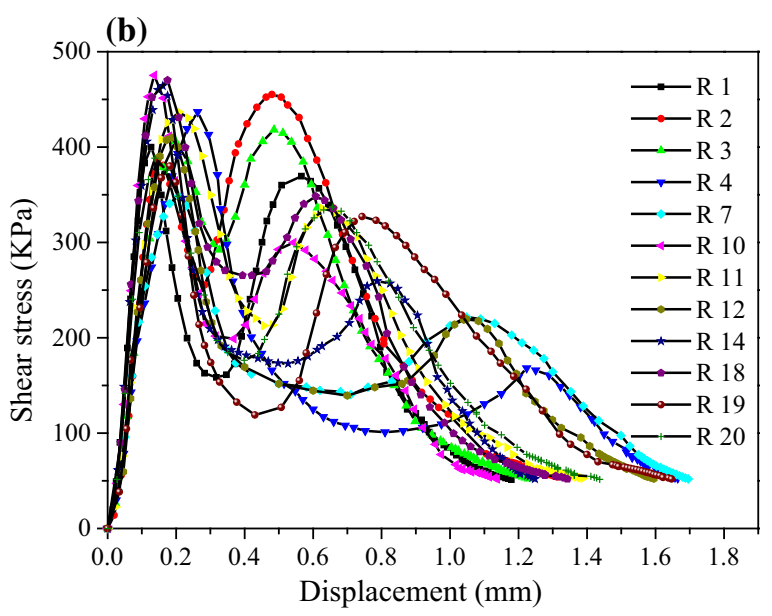

Fig. 2 Stress-displacement curves of experimental group (EG): a Single-peak curves (SPCs); b Double-peak curves (DPCs)

(a)

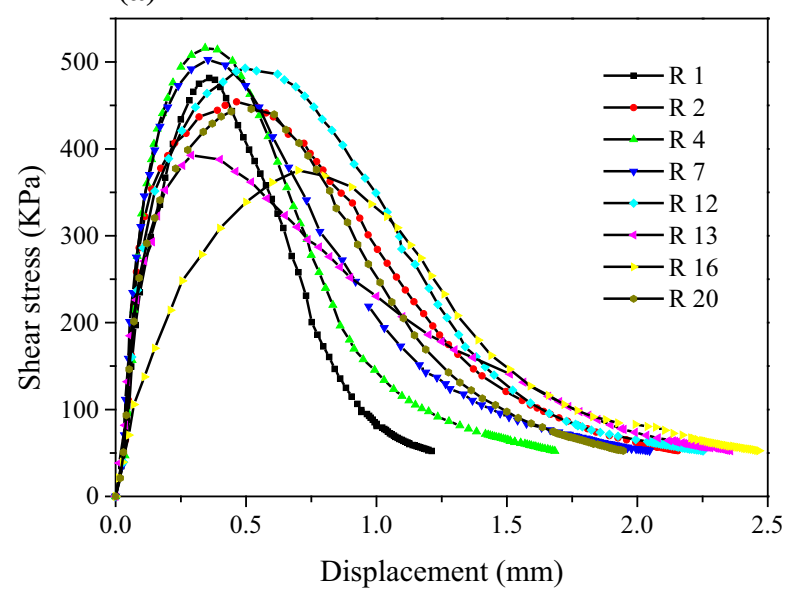

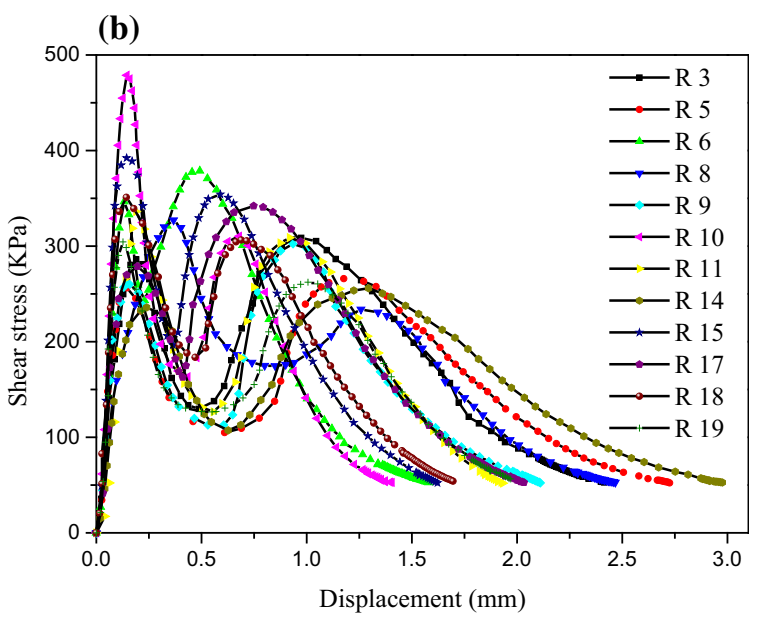

Fig. 3 Stress-displacement curves of control group of surface treatment (CGST): a Single-peak curves (SPCs); b Double-peak curves (DPCs)

stress-displacement curves which are shown in Figs. 2, 3, 4, 5 for all the experimental conditions. It exhibits that two kinds of the complete stressdisplacement curves, the familiar single-peak curves
(SPCs) and the first-known double-peak curves (DPCs), are observed. Each of the SPCs (or the DPCs) for all the experimental conditions follows a similar mode, respectively. 
(a)

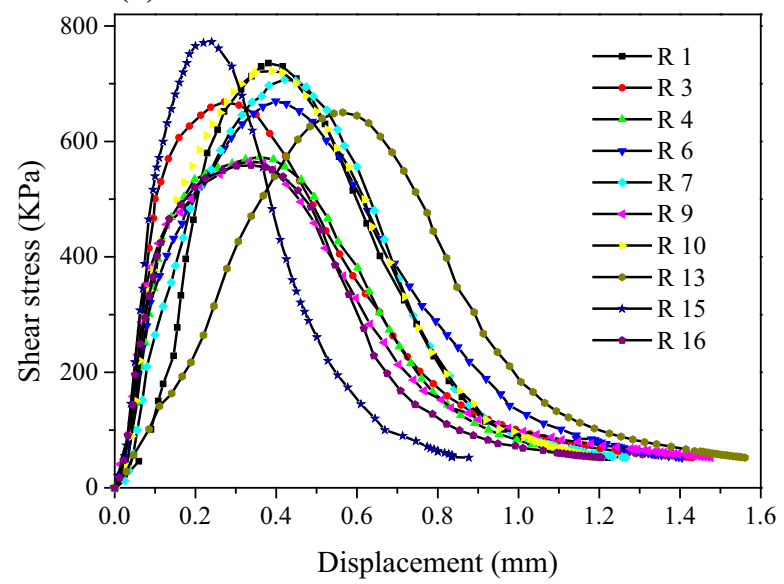

(b)

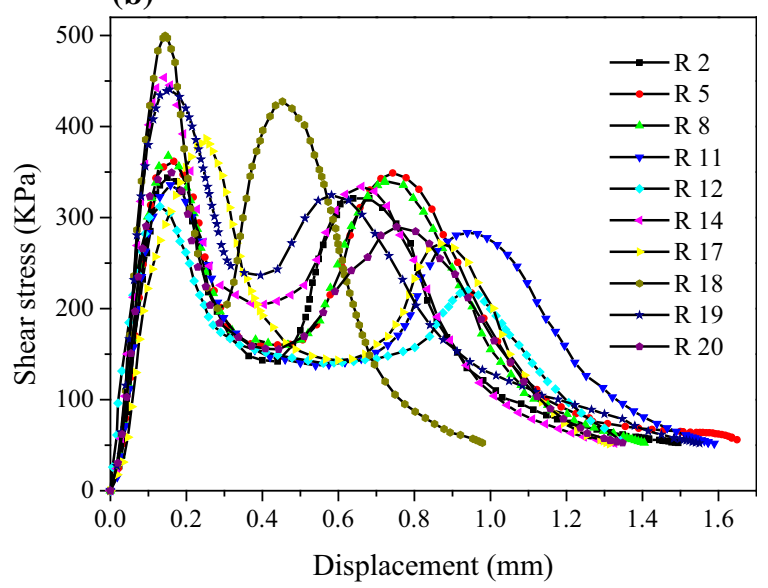

Fig. 4 Stress-displacement curves of control group of fabrication stress (CGFS): a Single-peak curves (SPCs); b Double-peak curves (DPCs)

(a)

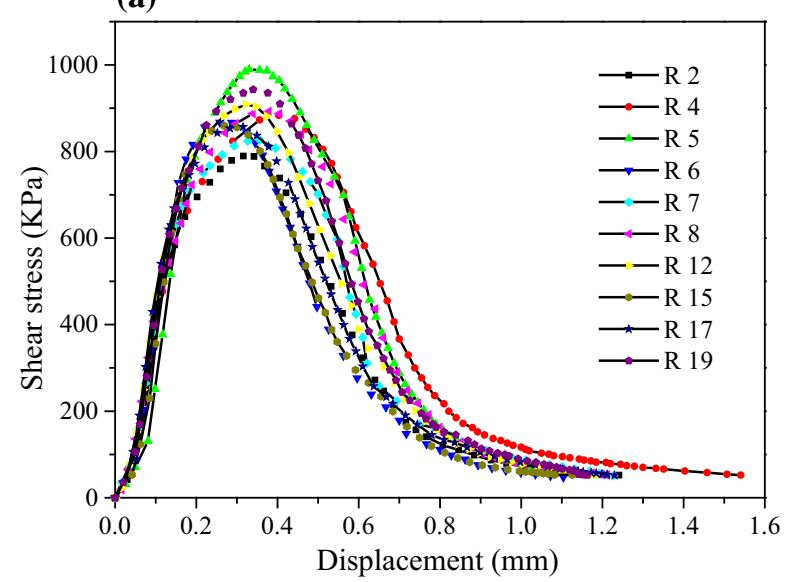

(b)

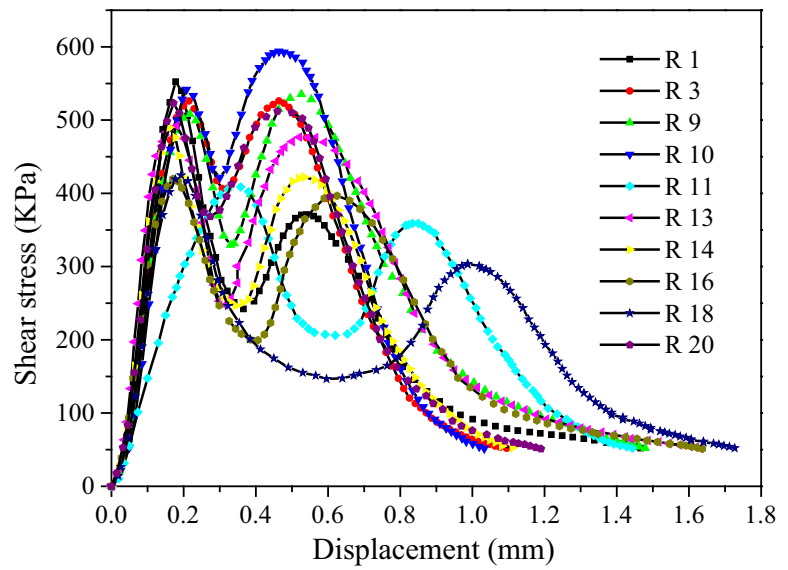

Fig. 5 Stress-displacement curves of control group of loading rate (CGLR): a Single-peak curves (SPCs); b Double-peak curves (DPCs)

The SPCs shown in Figs. 2, 3, 4, 5a can be seen in some researches $[5,14,19,21-23]$ : the shear stress increases initially and then decreases with increasing shear displacement. The ascending parts of the curves show an approximately linear shape before approaching to a peak value. The descending parts of the curves show a strain softening behavior until leveling out a plateau which is defined as the residual shear stress.

The DPCs shown in Figs. 2, 3, 4, 5b are first-known but obtained by a direct shear test method, which are analogous to the load-deflection curves by Canestrari et al. [24]. The shape of the DPC is considerably different from SPC but follows a similar mode among them for all the experimental conditions. Each of the
DPCs generates two peak values with increasing shear displacement. It implies that DPC may be due to the separation of adhesive failure from cohesive failure $[8,9,11,12]$. The whole curve generates the first-peak stress with converging on a displacement $\left(x_{1}\right)$, and then produces the second-peak stress with presenting stochastic displacement $\left(x_{3}\right)$ distribution (the specific positions of $x_{1}$ and $x_{3}$ is shown in Fig. 6). It is observed that the DPCs go through four distinct stages, including ascending part, descending part, reascending part and secondary descending part. The first stage corresponds to the linear elastic behavior of asphaltaggregate interface. In this case, since the interfacial bonding is the weakest link, which is caused by 
(a)

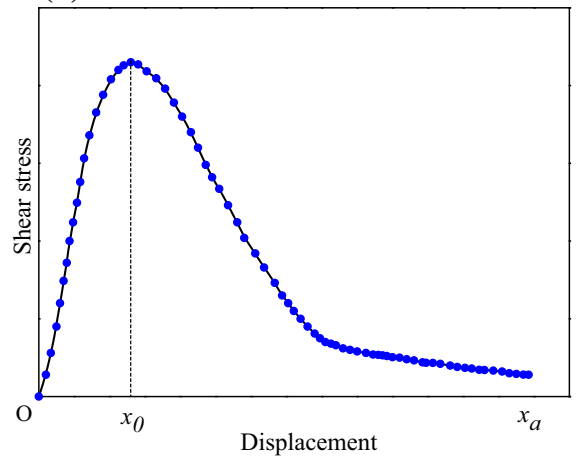

(b)

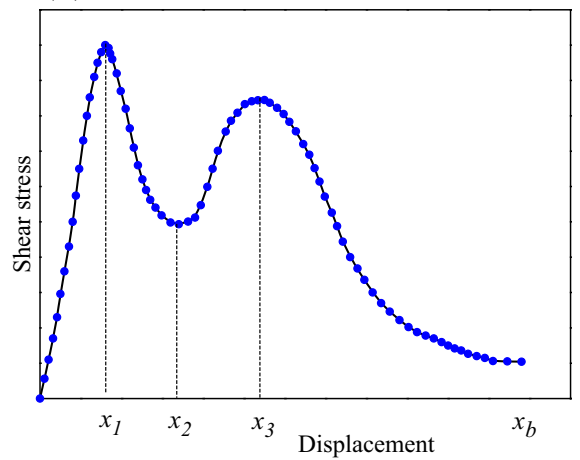

Fig. 6 Stress-displacement curves of sandwiched specimens under direct shear loading: a Single-peak curve (SPC); b Double-peak curve (DPC)

different theories $[16,25]$, the second stage corresponds to the interfacial failure between asphalt and aggregate. After a transition phase of the cohesiveadhesive hybrid failure, the third stage regarded as reascending part is due to the separation of molecules within the asphalt film and the shear stress increases again until reaching the second peak value. The forth stage regarded as secondary descending part corresponds to the failure process when the shear stress reaches an approximately constant value. The reason for these conclusions will be introduced in the following sections.

A direct comparison of the SPC and the DPC, a general trend exists that the peak stress of the SPC is higher than alternative peak stress of the DPC for each condition. On the one hand, the ascending parts of the SPCs almost overlap each other, while the post-peak curves are relatively stochastic in Figs. 2, 3, 4, 5a. On the other hand, the ascending parts of the DPCs also show approximately linear shapes and gather together, while the post curves including descending parts, reascending parts and secondary descending parts are remarkably different and complex under the same experimental condition, especially in the final two stages shown in Figs. 2, 3, 4, 5b. These differences are probably due to the friction at the asphalt-aggregate interface [19, 26, 27] and internal bonding condition [25]. It should be noted that the asphalt-aggregate interface and the molecules within the asphalt film can not be completely independent of each other [27]. Therefore, this paper suggests that the plastic flow, delay and viscoelastic properties of asphalt mastic can retard the secondary ascending parts so as to produce stochastic second-peak curves [27, 28]. In a word, due to the differences of the internal bonding of specimens and external loading process, each of the independent replicate specimens has the mutative capacity for shear resistance to some extent.

It also implies that the complete stress-displacement curves are variable for replicate specimens with the identical asphalt-aggregate materials under each experimental condition, which indicates that the mechanical behavior can be governed by the characteristics of the stone surface, asphalt film thickness, loading rate and other potential factors.

\subsection{Effect of stone surface treatment}

Evaluation and comparison of experimental group (EG) and control group of surface treatment (CGST), the effect of the methods of stone surface treatment on the mechanical behavior is investigated. The polished surface was created by polishing the saw-cut surface with a fine-polishing disk, and the sandblasted surface was made by blasting the saw-cut surface with a coarse-blasting disk. In Fig. 2a, the shear strengths of the specimens by means of polished treatment range from 553 to $746 \mathrm{kPa}$. The shear strengths of the specimens with sandblasted surface shown in Fig. 3a range from 375 to $516 \mathrm{kPa}$, which are approximately $70 \%$ of the shear strengths of the specimens with polished surface. Simultaneously, it is evident from Figs. 2, 3, 4, 3b that both of the first-peak stress and the second-peak stress of the DPCs reduce to a certain extent. 
It has revealed that the interfacial treatment of asphalt-aggregate specimens has an obvious effect on the interfacial bond strength. The micro-structure characteristics and the adhesive quality of specimen interface determine the experimental nature $[20,21]$. The classification of the surface treatment is based on the degree to which the interface is rough, smooth or polished. Surface texture depends on the degree to which the shear stress acting on the stone interface has smoothed or roughened it. The interfacial textures of specimen in interlocking situation significantly influence the shear strength and the bonding condition. Although the interfacial micro-structures of the experimental specimens were not measured, possibly a rougher surface texture results in a larger adhesive strength between the particles and asphalt at a specific level [21].

\subsection{Effect of asphalt film thickness}

Using two types of fabrication stresses can lead to two types of asphalt content, which are nearly equivalent to being two types of asphalt film thicknesses. Comparing of experimental group (EG) and control group of fabrication stress (CGFS), the shear strengths of the specimens subjected to fabrication stress of $89.9 \mathrm{kPa}$ range from 553 to $746 \mathrm{kPa}$ in Fig. 2a. In the same way, the shear strengths of the specimens subjected to fabrication stress of $22.5 \mathrm{kPa}$ shown in Fig. 4a range from 558 to $772 \mathrm{kPa}$ that mostly resemble those of the experimental group (EG). Likewise, Figs. $2 \mathrm{~b}$ and $4 \mathrm{~b}$ also indicate that the first/ second-peak stresses of the DPCs have no remarkable differences between the two groups.

Generally, it is believed that an optimum asphalt film thickness of about 9-10 microns is necessary to ensure durability and fatigue resistance of asphalt mixtures $[18,29]$. However, differences in the shear strength of the specimens with various asphalt film thicknesses are not obvious based on the results mentioned above. The idealized adhesive zone represented by two stone columns glued by a thin bitumen interlayer was appropriate to well explain the mechanical behavior of the interfacial adhesion, the transition phase from adhesion to cohesion and the completely cohesive failure. The research reveals that the bitumen film shows a peak strength value at 15-25 microns and thicker film will result in cohesive failure of bulk bitumen [17].
This may be due to the asphalt film thicknesses of two groups of analyzed specimens that were almost the same or possible with similar and random microstructures. Thus, two groups of comparative tests may not be adequate to evaluate the effect of asphalt film thickness. This paper also suggests that an exact range of asphalt film thickness for obtaining the DPC should be determined by conducting different asphalt film thickness tests.

\subsection{Effect of loading rate}

Comparing of experimental group (EG) and control group of loading rate (CGLR), a general trend expected exists that a larger loading rate implies a higher shear strength. In Fig. 2a, the shear strengths of the specimens at a rate of $5 \mathrm{~mm} / \mathrm{min}$ range from 553 to $746 \mathrm{kPa}$. The shear strengths of the specimens at a rate of $9 \mathrm{~mm} / \mathrm{min}$ shown in Fig. 5a range from 790 to $990 \mathrm{kPa}$, which are approximately $1.43-1.32$ times higher than those of the specimens at a rate of $5 \mathrm{~mm} /$ min. Furthermore, the first/second-peak stresses shown in Fig. 2b range from 348 to $474 \mathrm{kPa}$, 219-455 kPa, respectively. The first/second-peak stresses shown in Fig. 5b range from 408 to $552 \mathrm{kPa}, 303-593 \mathrm{kPa}$, which are much higher than those shown in Fig. 2b, respectively.

It can be seen that loading rate has a remarkable influence on the mechanical behavior of asphaltaggregate specimens, including the shear strengths of the SPCs, first/second-peak stresses and curve shape distribution of the DPCs. As mentioned above, these results agree well with previous findings that larger loading rate means that a larger adhesive strengths can be developed [2].

\subsection{Analysis of displacement}

In order to summarize the characteristics of the singlepeak curves (SPCs) and the double-peak curves (DPCs), the results including the average values of displacement and corresponding standard deviations for four groups of comparative tests are presented in Table 3.

These results are compared for several characteristics which basically reflect the shapes and boundary conditions of the SPCs and the DPCs. Some potential regularities that can be seen in Figs. 2, 3, 4, 5 and Table 3. Clearly, it confirms that $x_{0}$ and $x_{1}$ exhibit 
Table 3 The average values of the displacement ( $\mathrm{SD}$ in round brackets)

\begin{tabular}{lllll}
\hline Experimental condition & SPC & DPC & & \\
\cline { 2 - 5 } & $x_{0}(\mathrm{~mm})$ & $x_{1}(\mathrm{~mm})$ & $x_{2}(\mathrm{~mm})$ & $x_{3}(\mathrm{~mm})$ \\
\hline EG & $0.272(0.0378)$ & $0.172(0.0352)$ & $0.534(0.258)$ & $0.734(0.237)$ \\
CGST & $0.442(0.141)$ & $0.178(0.0592)$ & $0.491(0.181)$ & $0.843(0.262)$ \\
CGFS & $0.366(0.0776)$ & $0.162(0.0305)$ & $0.474(0.135)$ & $0.715(0.151)$ \\
CGLR & $0.300(0.0531)$ & $0.200(0.0554)$ & $0.325(0.164)$ & $0.500(0.172)$ \\
\hline
\end{tabular}

superior convergence behavior, and focus on a value with smaller standard deviations (SD), respectively. In contrast, $x_{2}$ and $x_{3}$ are much more stochastic and uncertain with much higher standard deviations (SD) compared to those of $x_{0}$ and $x_{1}$, respectively.

As shown in Fig. 6, $x_{0}$ is the average displacement of the shear strength of the SPC, $x_{1}$ is the average displacement of the first-peak stress of the DPC, $x_{2}$ is named as the average displacement of trough stress of the DPC and $x_{3}$ represents the average displacement of the second-peak stress of the DPC.

Figures 2, 3, 4, 5 show a summary that four groups of comparative tests are sensitive to different experimental procedures and the internal bonding conditions of specimens. Accordingly, the shear stressdisplacement relationship introduced in this study provides a better prediction for the shear mechanical behavior of asphalt-aggregate systems subjected to direct shear loading. More specifically, in view of the effects of experimental differences on the overall properties and the rather numerous results presented here, it is possible to imply that the shear failure mechanisms of asphalt-aggregate specimens are similar among the four groups of tests.

\section{Failure mechanism analysis}

Based on the experimental methodology and results elaborated above, failure mechanisms are presented and discussed in this section.

\subsection{Theoretical background}

It is generally believed that the single-peak curves (SPCs) can be obtained by compression test [14, 21], direct shear test [5, 19], and tensile test [22, 23]. Something is just the other way around, the experiment reported here has obtained the double-peak curves (DPCs), which have no significant background research in the literature. Thus, the existing studies for asphalt mixtures are inadequate to capture the complete stress-displacement curves during the direct shear tests.

The DPCs can not distinctly reflect the internal physical changes of asphalt-aggregate interface and the failure mechanisms. The failure has not been well understood and should be attributed to a wide variety of causes. The internal bonding condition of asphaltaggregate specimen and external loading process will have great influences on the failure mechanisms and mechanical behavior of the tested specimen. In particular, the interface between asphalt and aggregate with random microstructures, possibly with anisotropy and defects [30], should be taken into account more deeply in the experimental procedures and result analysis.

As a matter of fact, the adhesion between asphalt and aggregate is an important technical indicator, which directly relates to the stability and durability of asphalt mixture and also influences the quality of asphalt pavements [31]. According to the standard test method, there are three types of failure modes that can occur during direct shear tests: adhesion, cohesion and a combination of both these $[8,9,11,12]$. Furthermore, the shear fracture occurs through the asphaltaggregate interface as well as through the asphalt film.

Evidently, the direct shear test closely associates with the internal bonding condition of specimen, external loading process and testing machine. In response, the stress-displacement curve with the time-dependent properties shows nearly linear-elastic property at small deformation initially, the transition from adhesion to cohesion, and then complete failure with a large plastic deformation. Furthermore, this direct shear test method might also confirm the hybrid failure of adhesion and cohesion, especially when the obvious DPCs appeared. 


\subsection{Analysis of interface stress}

The reliability of the adhesive interface depends on the bonding condition and the internal stress. Different materials have different natures and the same materials also have various micro-structures. Thus, even if the asphalt-aggregate combinations are not subjected to external shear loading, the interface of asphaltaggregate specimen also bear much higher internal stress which is mainly caused by the following three aspects [25].

First, asphalt and aggregate have intrinsic thermal expansion coefficients and the various temperature would generate internal stress between asphalt and aggregate surface. Second, the different hygroscopic expansion coefficients also can produce some internal stress in the hot and humid environment. Finally, the poor interface with air voids and imperfect asphalt film also results in internal stress concentration.

A review of the literature proves that water affects the adhesive bond between asphalt and aggregate much more than the asphalt cohesion [8]. If a good bond exists between the binder and the aggregate, failure of the mixture occurs within the binder, but, if a poor bond exists, adhesive failure of the mixtures occurs at the binder-aggregate interface [32]. Some results from the fracture model, which was adopted to simulate the fracture damage within the Fine Aggregate Matrix (FAM), indicated that a very high level of stress concentrated near the aggregate tip that was close to another aggregate tip and crack initiated in the aggregate surface along with the development of FAM interface damage [33].

The aforementioned review indicates that the internal stress at the asphalt-aggregate interface during specimen preparation have a significant effect on the adhesive bond. For the sandwiched specimen, the asphalt-aggregate interface had born much more stress concentrations than the asphalt film before performing the shear test under the dry condition. Therefore, the asphalt-aggregate interface could be broken sharply first, and slightly delayed cohesive failure of the asphalt film happened in this direct shear test.

\subsection{Theory of rheological models}

Since the rheological properties of the asphalt-aggregate systems is shown by a direct shear test method, rheological models can be used to describe the rheological characteristics of the asphalt-aggregate systems. Considering the shear test, Kelvin's model [28], fits the shearing results due to its constitutive model and mathematical efficiency, and its constitutive equation is given as Eq. 2.

$\tau=G \gamma+\eta \dot{\gamma}$

where $\tau$ is the shear stress, $\gamma$ is the shear strain, $G$ is shear modulus and $\eta$ is the viscous parameter.

For the study of creep behavior, the function of Laplace transform [3] is shown as Eq. 3.

$\tilde{f}(s)=\int_{0}^{\infty} f(t) \mathrm{e}^{-s t} \mathrm{~d} t$

with $\tau=\tau_{0} \mathrm{H}(t)$, the constitutive equation can be written as

$\tau_{0} \mathrm{H}(t)=G \gamma+\eta \dot{\gamma}$

After applying the Laplace transform, the equation can be expressed as

$\frac{\tau_{0}}{s}=G \gamma(s)+\eta s \gamma(s)$

Then

$\gamma(s)=\frac{\tau_{0}}{s(G+\eta s)}$

Carrying out the Laplace transform again, the creep equation is obtained as

$\gamma(t)=\frac{\tau_{0}}{G}\left[1-\mathrm{e}^{-\frac{G}{\eta} t}\right]$

With

$t_{\mathrm{d}}=\frac{\eta}{G}$

$\gamma\left(t_{\mathrm{d}}\right)=0.63 \frac{\tau_{0}}{G}$

From Eq. 8, $t_{\mathrm{d}}$ is defined as the delay time of the viscoelastic asphalt mastic, and represents a physical coefficient of viscoelastic model. In Fig. 7, the intersection abscissa of the tangent line of the original point and the horizontal asymptote of the creep curve is considered as $t_{\mathrm{d}}$, which is equivalent to almost $63 \%$ of the time of elastic deformation [28]. Therefore, Kelvin's model is also called as a time-delay model for the viscoelastic asphalt mastic, and can prove the 


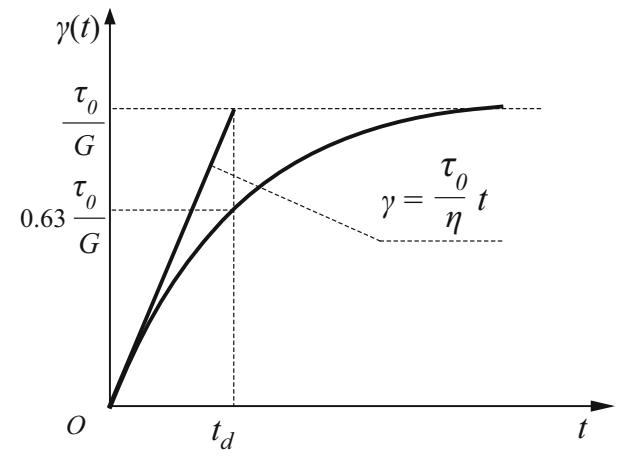

Fig. 7 Creep curve of Kelvin model

validity of the hysteresis for explaining the occurrence of the second-peak curves of the double-peak curves (DPCs).

Besides, the direct shear tests also have launched a review of micromechanical behavior of asphalt mixture. And the shear tests between asphalt mastic and aggregate can be expressed by a stiffness model of Liu et al. [27], which is built by combining a spring element [27] and Burger's model [3] in series with a friction parameter $\mu$ between them. What's more, aggregate is regarded as pure elastic material and the spring element that obeys Hooke's law is adopted to represent the constitutive mechanical behavior, Burger's model is employed to serve as the asphalt mastic [27].

Above all else, plastic shear flow exists in the asphalt film as well as at the asphalt-aggregate interface during the shear testing. The hysteretic nature of the viscoelastic asphalt mastic should be one of the reasons that the second-peak of the DPC appears in the direct shear test [28].

\subsection{Analysis and evaluation of the general test results}

The tested specimens are typical composites, whose constituents with different mechanical performance include limestone aggregate, asphalt and air voids [4, 10, 34]. However, the same limestone columns may have some differences in physical properties among them, and the fabricating process of specimens may also contribute to the non-uniform asphalt films. A research demonstrated that high void content accelerates crack initiation [35], which amounts to a great promotion for the distress of asphalt mixture
[29]. It can be concluded that the variety of asphalt film and limestone surface makes the independent replicate specimens present the randomness of mechanical behavior and shearing resistance.

In addition, the mechanical properties of asphalt include viscoelasticity and hysteresis [28]. According to the above mentioned, when the shear stress exceeds the critical resistance of asphalt-aggregate interface, the first-peak curve will be obtained. During the interfacial resistance towards collapse, the internal resistance from asphalt molecules not only show a short delayed phase but also keep the resistance simultaneously. Then, it rapidly evolves the secondpeak curve when the shear stress exceeds the critical resistance of asphalt film. It is worth indicating that the peak displacements of the second-peak curves of the DPCs are stochastic, which relate to the shear process.

Consequently, the clear separations shown in Figs. 2, 3, 4, 5b suggest that the first-peak curves of the DPCs should be regarded as adhesive failure, the second-peak curves of the DPCs should be defined as cohesive failure, and the complete shear process should be known as a combination of adhesivecohesive failure. The characteristics of the DPCs present transient-elasticity, viscoelasticity and nontransient viscoplasticity. The shear flow, viscosity and hysteresis of asphalt mastic might contribute to the delayed second-peak curves of the DPCs [28].

In conclusion, the findings of the direct shear tests are consistent with two intrinsic parts: asphalt binder test and asphalt-aggregate interfacial test. Results based on qualitative and quantitative analyses indicate that the potential failure modes include adhesive failure at the asphalt-aggregate interface and cohesive failure within the asphalt film.

\section{Conclusions and suggestions for later research}

This paper presents an experimental study on the shear characteristics, mechanical behavior of asphalt-aggregate systems by the direct shear test method under four different experimental conditions. The test results and the discussions presented in this paper allow the following main conclusions to be drawn:

(1) The proposed test method is effective for determining the shear characteristics of asphalt-aggregate systems, which is evident 
from the results and the experimental repeatability for four experimental conditions.

(2) Two kinds of the complete stress-displacement curves, including the general single-peak curve (SPC) and the first-known double-peak curve (DPC), are clearly observed. Each of the double-peak curves (DPCs) generates two peak values with increasing shear displacement, produces the first-peak stress with converging on a displacement, and then appears the secondpeak stress with presenting stochastic displacement distribution.

(3) The mechanical behavior of asphalt-aggregate systems can be governed by the characterizes of the stone surface, asphalt film thickness, loading rate and other unknown factors. Especially, the loading rate has a pronounced effect on the shear strengths of the SPC, the first-peak stress and the second-peak stress of the DPC.

(4) In the shearing case, it has been found that the double-peak curves (DPCs), which are presented here for the first time, have confirmed that the potential failure modes include adhesive failure at the asphalt-aggregate interface and cohesive failure within the asphalt film. Furthermore, the shear flow, viscosity and hysteresis of asphalt mastic might contribute to the delayed second-peak curves of the DPCs.

(5) Extending the current experimental research and trying to verify the analysis results (stress, displacement, failure mode and influence factor) with laboratory tests. And a rational range of asphalt film thickness for generating the double-peak curve (DPC) is necessary to confirm in later researches. Maybe there are some other unknown factors that also contribute to the direct shear test for obtaining the DPCs, which deserve the further research by factorial experiment design method and theory method.

Acknowledgements The authors gratefully acknowledge the funding support by the National Natural Science Foundation of China (No. 51408173). The authors also thank School of Automotive and Transportation Engineering, Hefei University of Technology for providing a laboratory.

\section{Compliance with ethical standards}

Conflict of interest The authors declare that they have no conflict of interest.
Open Access This article is distributed under the terms of the Creative Commons Attribution 4.0 International License (http:// creativecommons.org/licenses/by/4.0/), which permits unrestricted use, distribution, and reproduction in any medium, provided you give appropriate credit to the original author(s) and the source, provide a link to the Creative Commons license, and indicate if changes were made.

\section{References}

1. Andrea AD, Tozzo C (2016) Dynamic tests on bituminous layers interface. Mater Struct 49(3):1-12

2. Yan XL, Liang CY, Ai T, An SW (2014) Study on the viscoplastic model of the interfacial shear between bitumen and stone. Chin J Civ Eng 47(2):136-144

3. Gao YM, Dong MS, Li LL, Wang LN, Sun ZB (2015) Interface effects on the creep characteristics of asphalt concrete. Constr Build Mater 96:591-598

4. Dong MS, Gao YM, Li LL, Wang LN, Sun ZB (2016) Viscoelastic micromechanical model for dynamic modulus prediction of asphalt concrete with interface effects. J Cent South Univ 23:926-933

5. Yao B, Li FC, Wang X, Cheng G (2016) Evaluation of the shear characteristics of steel-asphalt interface by a direct shear test method. Int J Adhes Adhes 68:70-79

6. Liu Q, Xiao JZ, Sun ZH (2011) Experimental study on the failure mechanism of recycled concrete. Cem Concr Res 41:1050-1057

7. Airey GD, Hunter AE, Collop AC (2008) The effect of asphalt mixture gradation and compaction energy on aggregate degradation. Constr Build Mater 22:972-980

8. Canestrari F, Cardone F, Graziani A, Santagata FA, Bahia HU (2010) Adhesive and cohesive properties of asphaltaggregate systems subjected to moisture damage. Road Mater Pavement Des 11(sup. 1):11-32

9. Kanitpong K, Bahia H (2005) Relating adhesion and cohesion of asphalts to the effect of moisture on laboratory performance of asphalt mixtures. Transp Res Rec 1901:33-43

10. Zhang JZ, Airey GD, Grenfell JRA (2016) Experimental evaluation of cohesive and adhesive bond strength and fracture energy of bitumen-aggregate systems. Mater Struct 49(7):2653-2667

11. Xu GJ, Wang H (2016) Study of cohesion and adhesion properties of asphalt concrete with molecular dynamics simulation. Comput Mater Sci 112:161-169

12. Vencl A, Arostegui S, Favaro G, Zivic F, Mrdak M, Mitrovic S, Popovic V (2011) Evaluation of adhesion/cohesion bond strength of the thick plasma spray coatings by scratch testing on coatings cross-sections. Tribol Int 44:1281-1288

13. Lyne AL, Krivosheeva O, Birgisson B (2013) Adhesion between bitumen and aggregate: implementation of spectroscopic ellipsometry characterisation and estimation of Hamaker's constant. Mater Struct 46(10):1737-1745

14. Zhu HR, Sun L (2013) A viscoelastic-viscoplastic damage constitutive model for asphalt mixtures based on thermodynamics. Int J Plast 40:81-100

15. Liu YW, Apeagyei A, Ahmad N, Grenfell J, Airey G (2014) Examination of moisture sensitivity of aggregate-bitumen 
bonding strength using loose asphalt mixture and physicochemical surface energy property tests. Int J Pavement Eng 15(7):657-670

16. Mo LT, Huurman M, Wu SP, Molenaar AAA (2009) Ravelling investigation of porous asphalt concrete based on fatigue characteristics of bitumen-stone adhesion and mortar. Mater Des 30:170-179

17. Mo LT, Huurman M, Wu SP, Molenaar AAA (2011) Bitumen-stone adhesive zone damage model for the mesomechanical mixture design of ravelling resistant porous asphalt concrete. Int J Fatigue 33:1490-1503

18. Sengoz B, Agar E (2007) Effect of asphalt film thickness on the moisture sensitivity characteristics of hot-mix asphalt. Build Environ 42:3621-3628

19. Yin AY, Yang XH, Zeng GW, Gao H (2015) Experimental and numerical investigation of fracture behavior of asphalt mixture under direct shear loading. Constr Build Mater $86: 21-32$

20. Lent DQV, Molenaar AAA, Ven MFCVD (2009) Influence treatment in laboratory of stone surface on the surface roughness. J Test Eval 37(5):417-423

21. Hong L, Gu XL, Lin F (2014) Influence of aggregate surface roughness on mechanical properties of interface and concrete. Constr Build Mater 65:338-349

22. Valdés-Vidal G, Calabi-Floody A, Miró-Recasens R, Norambuena-Contreras J (2015) Mechanical behavior of asphalt mixtures with different aggregate type. Constr Build Mater 101:474-481

23. Mehrara A, Khodaii A (2013) A review of state of the art on stripping phenomenon in asphalt concrete. Constr Build Mater 38:423-442

24. Canestrari F, Belogi L, Ferrotti G, Graziani A (2015) Shear and flexural characterization of grid-reinforced asphalt pavements and relation with field distress evolution. Mater Struct 48(4):959-975

25. Zhang J (2011) Application of interface mechanics in interface stress and the cohesive zone model. Ch. 1 (Sec. 1). Zhengzhou University Press, Zhengzhou
26. Pasquini E, Giacomello G, Pasetto M, Canestrari F (2015) Laboratory evaluation of the effect of low-temperature application of warm-mix asphalts on interface shear strength. Constr Build Mater 88:56-63

27. Liu Y, Dai QL, You ZP (2009) Viscoelastic model for discrete element simulation of asphalt mixtures. J Eng Mech 135(4):324-333

28. Fan GQ (1993) Rheological mechanics of geotechnical engineering. Ch.3 (Sec. 4). China Coal Industry Publishing Home, Beijing

29. Topal A, Sengoz B (2005) Determination of fine aggregate angularity in relation with the resistance to rutting of hotmix asphalt. Constr Build Mater 19:155-163

30. Kandhal PS, Chakraborty S (1996) Effect of asphalt film thickness on short and long term aging of asphalt paving mixtures. NCAT report 96-01

31. Huet C (1999) Coupled size and boundary-condition effects in viscoelastic heterogeneous and composite bodies. Mater Struct 31(12):787-829

32. Yan XL, Liang CY (2001) Study of the shear adhesiveness between bitumen and rock. Chin J Highw Transp 14(2):25-28

33. Tarrer AR, Wagh V (1991) The effect of the physical and chemical characteristics of the aggregate on bonding. National Research Council, Washington (DC)

34. Wang H, Wang J, Chen JQ (2014) Micromechanical analysis of asphalt mixture fracture with adhesive and cohesive failure. Eng Fract Mech 132:104-119

35. Zhang XN, Yin YM, Zou GL (2010) Viscoelastic performance of asphalt mixture with different void contents. Chin J Highw Transp 23(4):1-7

36. Freitas ED, Pereira P, Picado-Santos L, Papagiannakis AT (2005) Effect of construction quality, temperature, and rutting on initiation of top-down cracking. Transp Res Rec 1929:174-182 